\title{
ON THE EXISTENCE OF REGULAR APPROXIMATE DIFFERENTIALS
}

\author{
ALBERT FADELL
}

ABSTRACt. We prove that for continuous real-valued functions on an open set in $n$-space, a sufficient condition for the existence a.e. of a regular approximate differential is that the functions have an ordinary total differential a.e. with respect to all but one variable.

1. Introduction. T. Radó ([4], [5]) and R. Caccioppoli and Scorza Dragoni [1] introduced the concept of regular approximate differential (Definition 3, below) and proved that in order for a continuous real-valued function defined on a planar open set to have a regular approximate differential a.e., it suffices that the function have first partial derivatives a.e. In this note we extend this result in a natural way to $n$-space by proving that, again for a continuous function on an open set, a sufficient condition for the existence a.e. of a regular approximate differential is the existence a.e. of a total differential with respect to all but one variable. This strengthens a result of Lodigiani [2], who required that the function have partial derivatives everywhere which are continuous with respect to all but one variable.

2. Notation, definitions and basic theorems. Let $p=\left(p^{1}, \cdots, p^{n}\right)$ denote points in $n$-space $R^{n}$, and $\|p-q\|=\left[\sum_{j=1}^{n}\left(p^{j}-q^{j}\right)^{2}\right]^{1 / 2}$ the distance between $p$ and $q$. We use $\nabla f(p)$ for the $n$-tuple of partial derivatives $\left(f_{1}^{\prime}(p), \cdots\right.$, $\left.f_{n}^{\prime}(p)\right)$ and set

$$
\nabla f(p) \cdot(p-q)=\sum_{j=1}^{n} f_{j}^{\prime}(p)\left(p^{j}-q^{j}\right) .
$$

The notation $m S$ will denote $n$-dimensional Lebesgue measure of a set $S$ in $R^{n}$.

Definition 1. Given a real-valued function $f: S \rightarrow R$ on a subset $S$ of $R^{n}$, suppose $f$ has partial derivatives at a point $p$ in $S$. Let

$$
e(p, q)=\frac{f(q)-f(p)-\nabla f(p) \cdot(q-p)}{\|q-p\|}, \quad p \neq q .
$$

Received by the editors July 17, 1971 and, in revised form, June 16, 1972. AMS (MOS) subject classifications (1970). Primary 26A54; Secondary 28A15. Key words and phrases. Regular approximate differential, total differential. 
Then $f$ is said to have a total differential (in the Stolz sense) at $p$ if $\lim _{q \rightarrow p} e(p, q)=0$.

DEFINITION 2. Given any point $p$ in $R^{n}$ let $\mathscr{C}(p)$ denote any family of oriented $n$-cubes (edges parallel to the coordinate axes) such that (i) $p$ is the center of each $n$-cube of $\mathscr{C}(p)$, and (ii) $p$ is a point of density for $U$ fr $C$, $C \in \mathscr{C}(p)$, where fr $C$ denotes the frontier of $C$. We term $\mathscr{C}(p)$ a thick regular family of $n$-cubes at $p$.

The following lemma is easily verified.

LEMMA 1. If $p$ is a point of (linear) density of a subset $S$ of $R^{n}$ in the direction of every coordinate axis, then there exists a thick regular family of $n$-cubes $\mathscr{C}(p)$ such that the lines through $p$ parallel to the coordinate axes intersect the faces of each $n$-cube in a point of $S$.

Definition 3. Given a real-valued function $f: S \rightarrow R$, where $S$ is a subset of $R^{n}$, we say that $f$ has a regular approximate differential at a point $p$ in $S$ if there exists a thick regular family $\mathscr{C}(p)$ such that $f$ restricted to $\cup(S \cap \mathrm{fr} C), C \in \mathscr{C}(p)$, has a total differential at $p$.

LEMMA 2. Let $p$ be a point of density of a measurable set $S$ in $R^{n}$. Then for every $\eta>0$ there exists a $\delta>0$ such that whenever $\|p-q\|<\delta$ for a point $q$ in $R^{n}$ there corresponds a point $q_{*}$ in $S$ satisfying the inequality $\left\|q-q_{*}\right\|<$ $\eta\|p-q\|$.

Proof. This is a simple exercise in the definition of density, and may be found implicitly in Rademacher [3].

COROLlaRY. Let $S$ be a bounded measurable set in $R^{n}$, and let $\eta>0$ be given. Then for every $\varepsilon>0$ there corresponds a closed subset $F$ of $S$ and $a$ number $\delta>0$ for which the following two properties hold:

(i) $m(S-F)<\varepsilon$.

(ii) For every $p$ in $F, q$ in $R^{n}$ with $\|p-q\|<\delta$ and $p^{k}=q^{k}$ for some integer $k$, there corresponds a point $q_{*}$ in $S$ such that $q_{*}^{k}=q^{k}$ and $\left\|q_{*}-q\right\|<$ $\eta\|p-q\|$.

Proof. This follows easily from Lemma 2 by applying it to $(n-1)$ dimensional sections of $S$ and choosing the set $F$ to uniformize the $\delta$.

LEMMA 3. Let $f: S \rightarrow R$ be a continuous real-valued function defined on a bounded open set $S$ with the following property: for almost every point $p \in S$ there exist $\delta>0, M>0$ such that

$\left(^{*}\right)|f(p)-f(q)| \leqq M\|p-q\|$ whenever $q \in S,\|p-q\|<\delta$ and $p^{k}=q^{k}$ for some $k$.

Then for every $\varepsilon>0$ there exists a measurable subset $E$ of $S$, and numbers $\delta>0, M>0$ such that (1) $m(S-E)<\varepsilon$, (2) for every $p \in E$ condition $(*)$ in the hypothesis holds. 
Proof. Define the set $E_{m}$ to consist of all points $p$ in $S$ such that $|f(p)-f(q)| \leqq m\|p-q\|$ if $q \in S,\|p-q\|<1 / m$ and $p^{k}=q^{k}$ for some $k$. Clearly, $E_{m}$ is an ascending sequence of sets with $m\left(S-\cup_{m} E_{m}\right)=0$ in view of the hypothesis. To show that each $E_{m}$ is closed relative to $S$ let $p_{n}$ denote any sequence of points of $E_{m}$ converging to $p_{0} \in S$. Suppose $q \in S, q^{k}=p^{k}$, and $\left\|p_{0}-q\right\|<1 / m$. Then, for $n$ large enough, $\left\|p_{n}-q\right\|<1 / m$. Let $\bar{p}_{n}$ denote the point such that $\bar{p}_{n}^{k}=q^{k}=p^{k}$, but $\bar{p}_{n}^{j}=p_{n}^{j}$ for $j \neq k$. Then, for $n$ large enough, $\bar{p}_{n} \in S$ since $S$ is open, and so $\left|f\left(\bar{p}_{n}\right)-f(q)\right| \leqq m\left\|\bar{p}_{n}-q\right\|$. Since $f$ is continuous on $S$ and clearly $\bar{p}_{n} \rightarrow p_{0}$ we infer that

$$
\left|f\left(p_{0}\right)-f(q)\right| \leqq m\left\|p_{0}-q\right\|
$$

and so $p_{0} \in E_{m}$, and the proof that $E_{m}$ is closed relative to $S$ is complete. Thus for any assigned $\varepsilon>0$ there is an $m_{0}$ such that $m\left(S-E_{m_{0}}\right)<\varepsilon$. We therefore take $E=E_{m_{0}}, \delta=1 / m_{0}$ and $M=m_{0}$, and the proof is complete.

\section{Main theorem.}

THEOREM. Let $f: S \rightarrow R$ be a continuous real-valued function defined on an open bounded set $S$ in $R^{n}$. Assume that $f$ has a total differential a.e. in the direction of every $(n-1)$-dimensional coordinate hyperplane. Then $f$ has a regular approximate differential a.e. on $S$.

Proof. Let $\varepsilon>0, \gamma>0$ be given arbitrarily. In view of the hypotheses of the theorem, the conditions of Lemma 3 are satisfied. Select a set $E=E_{1}$ and numbers $\delta=\delta_{1}$ and $M=M_{1}$, satisfying the conclusion of Lemma 3 . By hypothesis the partial derivatives exist a.e. on $S$ and hence by the wellknown theorem of Stepanoff [7], there exists a measurable subset $E_{2}$ of $E_{1}$ such that $f$ has a total differential at each point of $E_{2}$ with respect to $E_{2}$ and $m\left(E_{1}-E_{2}\right)<\varepsilon$. By Lusin's theorem we may assume without loss that $E_{2}$ is closed and the partial derivatives are continuous on $E_{2}$, hence $|\nabla f(p)| \leqq$ $M_{2}$ for some constant $M_{2}$ and every $p \in E_{2}$. Further, let $E_{3}$ denote the set of all points of $E_{2}$ which are points of density of $E_{1}$ in the direction of every coordinate axis and in the direction of every $(n-1)$-dimensional coordinate hyperplane. Then $m\left(E_{2}-E_{3}\right)=0$ (see Saks [6, p. 298] for the linear case). Finally, let $E_{4}$ be (see corollary of Lemma 2) a measurable subset of $E_{3}$ and let $\delta_{2}>0$ chosen so that

(i) $m\left(E_{3}-E_{4}\right)<\varepsilon$,

(ii) for every $p \in E_{4}$ and $q \in S$ with $\|p-q\|<\delta_{2}$ and $p^{k}=q^{k}$ for some $k$, there corresponds a $q_{*} \in E_{1}$ such that

$$
\left\|q-q_{*}\right\|<\frac{\gamma}{3 M}\|p-q\| \& q_{*}^{k}=q^{k}
$$

where $M=\max \left(M_{1}, M_{2}\right)$. 
Consider now any point $p_{0} \in E_{4}$, and let $\delta_{3}>0$ be chosen so that

$$
\begin{aligned}
\left|f(\bar{q})-f\left(p_{0}\right)-\nabla f\left(p_{0}\right) \cdot\left(\bar{q}-p_{0}\right)\right| \leqq(\gamma / 6)\left\|\bar{q}-p_{0}\right\| \\
\quad \text { when }\left\|\bar{q}-p_{0}\right\|<\delta_{3}, \bar{q} \in E_{2} .
\end{aligned}
$$

Take now any point $p_{0} \in E_{4}$. Let $\mathscr{C}\left(p_{0}\right)$ denote a thick regular family of $n$-cubes centered at $p_{0}$ such that (see Lemma 1) the lines through $p_{0}$ parallel to the coordinate axes intersect the frontiers of the $n$-cubes in points of $E_{3}$. Let $\delta=\min \left(\delta_{1}, \delta_{2}, \delta_{3}\right)$ and take any point $g \in S$ on the frontier of any $n$ cube $C$ of $\mathscr{C}\left(p_{0}\right)$ having diagonal-length less than $\delta$. Suppose without loss of generality that the 1 -axis is perpendicular to the face whereon $q$ lies. Then $q=\left(p_{0}^{1}+h, q^{2}, q^{3}, \cdots, q^{n}\right)$ for some number $h$. Let $p_{1}=\left(p_{0}^{1}+h\right.$, $\left.p_{0}^{2}, \cdots, p_{0}^{n}\right)$. Then $p_{1} \in E_{3}$, so that since $\left\|q-p_{1}\right\|<\delta_{2}$ we have a $q_{*}=$ $\left(p_{0}^{1}+h, q_{*}^{2}, \cdots, q_{*}^{n}\right) \in E_{1}$ satisfying (1). Then $\left\|q-q_{*}\right\|<\delta_{1}$ and $\left|q_{*}-p_{0}\right|<\delta_{3}$, and so we have the following sequences of inequalities:

$$
\begin{aligned}
\mid f(q) & -f\left(p_{0}\right)-\nabla f\left(p_{0}\right) \cdot\left(q-p_{0}\right) \mid \\
\leqq & \left|f(q)-f\left(q_{*}\right)\right|+\left|f\left(q_{*}\right)-f\left(p_{0}\right)-\nabla f\left(p_{0}\right) \cdot\left(q-p_{0}\right)\right| \\
\leqq & M_{1}\left\|q-q_{*}\right\|+\left|f\left(q_{*}\right)-f\left(p_{0}\right)-\nabla f\left(p_{0}\right) \cdot\left(q_{*}-p_{0}\right)\right| \\
& \quad+|\nabla f(p)| \cdot\left\|q-q_{*}\right\| \\
\leqq & M_{1}\left(\gamma / 3 M_{1}\right)\left\|q-p_{1}\right\|+(\gamma / 6)\left\|q_{*}-p_{0}\right\|+M_{2}\left(\gamma / 3 M_{2}\right)\left\|q-p_{1}\right\| \\
\leqq & (\gamma / 3)\left\|q-p_{0}\right\|+(\gamma / 3)\left\|q-p_{0}\right\|+(\gamma / 3)\left\|q-p_{0}\right\|=\gamma\left\|q-p_{0}\right\| .
\end{aligned}
$$

Thus, $f$ has a total differential at $p_{0}$ relative to the part of $S$ in $\bigcup$ fr $C$, $C \in \mathscr{C}\left(p_{0}\right)$, and so $f$ has a regular approximate differential at $p_{0}$. Since $p_{0}$ is an arbitrary point of $E_{4}$ and $m\left(S-E_{4}\right)<3 \varepsilon$, the theorem is proved.

\section{REFERENCES}

1. R. Caccioppoli and G. Scorza Dragoni, Necessità della condizione di Weierstrass per la semicontinuità di un integrale sopra una data superficie, Reale Acad. Ital. Mem. Cl. Sci. Fis. Math. Natur. 4 (1938), 251-268.

2. B. Lodigiani, Sulla differenziabilità asintotica regolare delle funzioni di piu variabili, Rend. Sem. Mat. Univ. Padova 22 (1953), 251-257. MR 15, 299.

3. H. Rademacher, Ueber partielle und totale Differenzierbarkeit. I, Math. Ann. 79 (1919), 340-359.

4. T. Rado, On the derivative of the Lebesgue area of continuous surfaces, Fund. Math. 30 (1938), 34-39.

5. - On absolutely continuous transformations in the plane, Duke Math. J. 4 (1938), 219-220.

6. S. Saks, Theory of the integral, 2nd ed., Monografie Mat., vol. 7, PWN, Warsaw, 1937.

7. W. Stepanoff, Sur les conditions de l'existence de la différentielle totale, Mat. Sb. 32 (1925), 511-526.

Department of Mathematics, State University of New York at Buffalo, AMHERST, NEW YORK 14226 